\title{
Manifolk: A 3D t-SNE Visualizer
}

\author{
Krishna Chaitanya Kodur \\ kck8298@mavs.uta.edu \\ University of Texas at Arlington \\ Arlington, Texas, USA
}

\author{
Ashwin Ramesh Babu \\ ashwin.rameshbabu@mavs.uta.edu \\ University of Texas at Arlington \\ Arlington, Texas, USA
}

\author{
Fillia Makedon \\ makedon@uta.edu \\ University of Texas at Arlington \\ Arlington, Texas, USA
}

\begin{abstract}
Manifolk is a tool to visualize the output of dimensionality reduction algorithms like t-SNE, PCA etc. One of this tool's main uses is that it de-clutters graphs by plotting data points pertaining to a subset of labels. The subset of labels to be plotted can be selected using the provided checkboxes. A case study on data from a publicly available action recognition dataset like UCF101 shows how this tool can help find outliers. With the rise in self-supervised methods for training deep neural networks, this tool helps researchers better visualize the embeddings learned by the model.
\end{abstract}

\section{KEYWORDS}

Deep Neural Networks, t-SNE, Clustering

\section{ACM Reference Format:}

Krishna Chaitanya Kodur, Ashwin Ramesh Babu, and Fillia Makedon. 2021. Manifolk: A 3D t-SNE Visualizer. In The 14th PErvasive Technologies Related to Assistive Environments Conference (PETRA 2021), June 29-7uly 2, 2021, Corfu, Greece. ACM, New York, NY, USA, 2 pages. https://doi.org/10.1145/ 3453892.3466619

\section{INTRODUCTION}

With many self-supervised learning frameworks [5] being proposed, there is a need to evaluate the performance of these frameworks for a downstream task such as classification, object detection, etc. A deep neural network (DNN) like ResNet[4] without a final classification layer, also called as an encoder, can be pretrained using the aforementioned frameworks. The output of the encoder can be termed as model embeddings. Following the pretraining, the next step is to add the final layer to the encoder based on the problem being solved and the whole network is trained again. This training is different from self-supervised training because, in this training, the encoder network is frozen i.e., the parameters in the encoder network are kept constant, thus only training the newly-added final layer. This step is called as finetuning. Only after finetuning the model performance metrics like accuracy and F1-score are calculated. In cases where the model is being trained on large datasets the pretraining can take considerable amount of time. Hence there is a need for visualizing how the model is performing during the pretraining. One method to visualize how the model is performing, is by plotting the output of the $\mathrm{t}$-distributed stochastic neighbor embedding ( $\mathrm{t}$-SNE) algorithm. Morever the input to $\mathrm{t}-\mathrm{SNE}$ are the

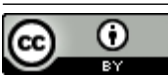

This work is licensed under a Creative Commons Attribution International 4.0 License.

PETRA 2021, June 29-fuly 2, 2021, Corfu, Greece

(C) 2021 Copyright held by the owner/author(s).

ACM ISBN 978-1-4503-8792-7/21/06

https://doi.org/10.1145/3453892.3466619 model embeddings hence there is no need for a final layer. t-SNE [8] is a dimensionality reduction algorithm that maps high-dimensional data which are model embeddings in our case into three dimensions (referred to as t-SNE embedding), which can then be subsequently plotted. A data point is an input to the DNN. A data point can be image or a video and it depends on the dataset using which the DNN is being trained upon. A dataset built for training DNN for classification has an associated ground truth label for each data point. If the model performs well, then the t-SNE plot should have data points from the same label clustered together; else, if the data points from the same label appear dispersed, i.e., not close together, that means that the model does not perform well. The t-SNE graph is helpful in visualizing the representation of the data that are learned during the training. The intuition is that a trained model with good accuracy encourages data points from the same or similar labels to be clustered together. The formation of clusters can be subjective as it is not quantitative, but observing the t-SNE over the DNN's training progression can indicate whether the model can approximate the data well. One of the drawbacks with python graphing libraries like matplotlib/seaborn/Plotly is that if t-SNE embeddings from many data points are plotted, they overshadow each other making the plots look cluttered. To address this Manifolk tool is proposed. The rest of the paper is organized in the following way: Section 2 explains related works where other dimensionality reduction algorithms similar to t-SNE are discussed. Other GUI's similar to Manifolk GUI and their drawbacks are also discussed. Section-3 explains Manifolk GUI in detail, along with a test case on how the GUI is useful. Section-4 explains the conclusion and future work.

\section{RELATED WORK}

One of the inspirations for the Manifolk GUI is the Dash t-SNE explorer app [2], and Embeddings Projector [1] by Tensorboard. The specific labels can be interactively selected in both the dash app and embeddings projector, and only those labels are plotted. One drawback is that only a single label or all the labels can be selected in Dash t-SNE explorer. However, in embeddings projector, multiple labels to be visualized can be selected, but for each small change in settings, e.g., changing the labels to be visualized, the projector starts rerunning the $\mathrm{t}-\mathrm{SNE}$ algorithm. However, rerunning the algorithm is not required as it can be run once according to the user preference. The proposed Manifolk GUI addresses all of these drawbacks.

\section{MANIFOLK}

There are many dimensionality reduction algorithms apart from t-SNE like Principal Component Analysis (PCA), Uniform Manifold Approximation and Projection (UMAP) [6], etc. The Manifolk GUI 


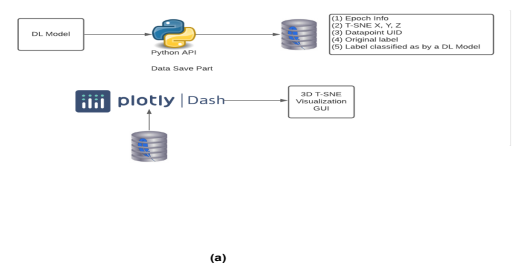

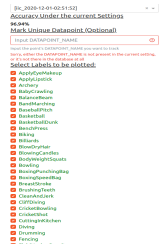

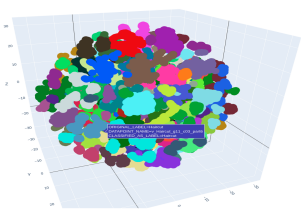

(b)

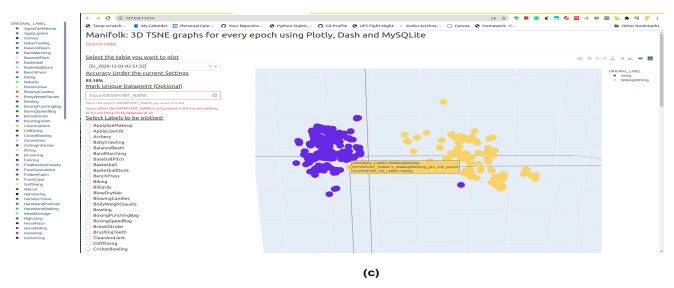

(c)

Figure 1: (a) Architecture diagram of Manifolk (b) Cluttered t-SNE graph (c) Outlier visualization case study

is explicitly designed, keeping the t-SNE embeddings in mind. However, embeddings from other dimensionality reduction techniques, e.g., UMAP, can also be used with the API as long as the embeddings generated by the algorithm for each data point have three coordinates that can be plotted on the graph. The architecture of the Manifolk tool as shown in Fig 1a is as follows: The API requires the following parameters to successfully plot the t-SNE embeddings: i.e., (i) epoch, (ii) t-SNE embeddings, (iii) Original label of the input, (iv) Unique identifier (UID) of the datapoint. For example if the data point corresponds to an image it's file name can be used as it's UID., and (v) Predicted label of the input (Optional) These parameters is saved to an SQLite database during training. The Manifolk GUI uses the saved data from the SQLite database to render the t-SNE plot. An example of the Manifolk GUI is shown in Figure 1b. Below are the features of the Manifolk GUI: i.e., (i) Ability to plot data points from selected labels only. Labels can be selected or deselected with the help of checkboxes provided in the GUI. This can especially help in de-cluttering the graph and visualizing only a subset of all labels., (ii) Provide extra details like UID on hovering over a data point. This feature is handy when finding more information about the outlier data points., (iii) Mark an individual datapoint using a unique marker to keep track of it. This feature is useful when a specific data point needs to be tracked during the training., and (iv) Provide a seek bar at the bottom to change the epochs' number to visualize the $\mathrm{t}$-SNE cloud at that specific epoch. This feature can be used to visualize the progression of the training. In the starting epochs, the clusters might not be formed, but in the later epochs, the clusters would have formed well given that the model has learned the representation of the data well.

In the Manifolk GUI, unique color is assigned to each unique true label, and that is how each data point is colored. Passing of the predicted label is optional, but the accuracy in the Manifolk GUI is calculated using the formula if they are passed: Let $\mathrm{x}$ be the number of data points from the selected labels whose true label and predicted label are the same and y be the number of data points from all the selected labels, then the accuracy would be $\frac{x}{y} \times 100 \%$.

To demonstrate how the Manifolk tool is useful when plotting $\mathrm{t}$-SNE of large datasets, a case study is performed using a model trained on the UCF101 dataset. UCF101[7] is a dataset, where there are 101 labels and a total of 13320 data points. Out of which 3696 are separated and used as test dataset. Fig $1 \mathrm{~b}$ shows the Manifolk GUI with all the data points from test dataset plotted. As there are many data points it looks cluttered. Finding outliers: the data points whose ground truth label and predicted label are different, would be difficult as the plot is cluttered. But by using Manifolk GUI, outliers can be easily found by de-cluttering the graph ( selecting only specific labels of interest from the checkboxes provided). During testing, augmented clips of 16 frames (hyperparameter) were generated from the data sample, which was the input to the model. In the Manifolk GUI in Figure 1c, all the violet points correspond to the groundtruth label "Skiing" and all the yellow points correspond to the groundtruth label "Walking with Dog". A yellow point is close to the violet cluster, which can be considered an outlier with respect to the yellow cluster. Its original label is "Walking with Dog" and its predicted label is "Skiing". On hovering over the yellow point, it was found that it was a clip obtained from v_WalkingDog_g11_c05, and when the clip was further analyzed, the clip did not contain any frames with a man or a dog walking. Hence, it was far away from the yellow cluster. The Manifolk GUI makes it easy to visualize and get insight into such details.

\section{CONCLUSION AND FUTURE WORK}

Manifolk is a tool that comprises a python API used to save t-SNE embeddings to an SQLite database which are subsequently used by the Manifolk GUI for visualization. The main use of this GUI is to declutter the graphs by visualizing a limited number of data points pertaining to only a certain class. This GUI can also help in easily visualizing the outliers as mentioned in $\S 3$. The future work is to convert Manifolk into a tensorboard plugin so that all the metrics are in one place for ease of use. The manifolk is available on GitHub [3].

\section{REFERENCES}

[1] [n.d.]. Embeddings Projector by Tensorboard.

[2] [n.d.]. Plotly Dash T-SNE on MNIST Data. https://dash-gallery.plotly.host/dashtsne/

[3] [n.d.]. TSNE GUI Github. https://github.com/krishnachaitanya7/Manifolk

[4] Kaiming He, Xiangyu Zhang, Shaoqing Ren, and Jian Sun. 2015. Deep Residual Learning for Image Recognition. arXiv:1512.03385 [cs.CV]

[5] Ashish Jaiswal, Ashwin Ramesh Babu, Mohammad Zaki Zadeh, Debapriya Banerjee, and Fillia Makedon. 2021. A survey on contrastive self-supervised learning. Technologies 9, 1 (2021), 2.

[6] Leland McInnes, John Healy, and James Melville. 2020. UMAP: Uniform Manifold Approximation and Projection for Dimension Reduction. arXiv:1802.03426 [stat.ML]

[7] Khurram Soomro, Amir Roshan Zamir, and Mubarak Shah. 2012. UCF101: A Dataset of 101 Human Actions Classes From Videos in The Wild. Technical Report. arXiv:1212.0402v1 http://crcv.ucf.edu/data/UCF101.php

[8] Laurens Van Der Maaten and Geoffrey Hinton. 2008. Visualizing Data using $t$-SNE. Technical Report. 2579-2605 pages. 